\title{
MODELAGEM HIDRODINÂMICA DE REATOR EGSB COM E SEM ADIÇÃO DE ZEÓLITA
}

\author{
G. T. CORREIA ${ }^{1}$, T. P. PÉREZ ${ }^{2}$, I. P. REYES ${ }^{3}$, M. ZAIAT $^{4}$, W. H. KWONG ${ }^{1}$ \\ ${ }^{1}$ Universidade Federal de São Carlos, Departamento de Engenharia Química \\ ${ }^{2}$ Instituto de Ciencia Animal, Cuba \\ ${ }^{3}$ Instituto Superior Politécnico José A. Echeverría, Centro de Inginiería de Procesos, Cuba \\ ${ }^{4}$ Escola de Engenharia de São Carlos - USP, Departamento de Hidráulica e Saneamento \\ E-mail para contato: gle_qca@hotmail.com
}

\begin{abstract}
RESUMO - Na literatura, a maioria dos trabalhos considera a hidrodinâmica dos reatores EGSB como mistura completa. O objetivo deste trabalho foi estudar mais detalhadamente a hidrodinâmica do EGSB, além de verificar a influência da adição de zeólita, aluminosilicato que, entre outras características, apresenta a possibilidade de acelerar o processo de partida de biorreatores. O volume do reator foi 3,04 L, TDH $12 \mathrm{~h}$, vazões de entrada $4 \mathrm{~mL} \cdot \mathrm{min}^{-1}$ e de recirculação 200 mL.min ${ }^{-1}$, zeólita natural (diâmetro médio $0,15 \mathrm{~mm}$ ). Realizaram-se três ensaios: somente água (E1), 24g de zeólita (E2) e 72g de zeólita (E3). Foi utilizado o método de pulso com traçador dextrana azul. Em estudo preliminar, os dados foram normalizados e aplicados nos modelos de tanques em série, dispersão de pequena e grande intensidade e mistura completa. Visando maior detalhamento e considerando as diferenças dimensionais entre tubo e separador, o reator foi dividido em dois reatores tubulares em série e o Peclet foi ajustado utilizado o método dos mínimos quadrados. No estudo preliminar, os dados apresentaram similaridade aos modelos de mistura completa e tanques em série. No modelo de dois reatores tubulares em série, com o ajuste do Pe, a região do tubo apresentou comportamento de reator tubular com alta dispersão $(\mathrm{Pe} \approx 5)$ e na região do separador apresentou comportamento de reator de mistura completa $(\mathrm{Pe} \approx 0)$. A adição de zeólita ao reator praticamente não causou influencia no escoamento.
\end{abstract}

\section{INTRODUÇÃO}

Os reatores EGSB (Expanded Granular Sludge Bed) surgiram como aperfeiçoamento dos UASB (Upflow Anaerobic Sludge Blanket), buscando diminuir efeitos de zonas mortas, fluxos preferenciais, curtos circuitos, entre outros problemas observados na prática nos reatores UASB (Kato et al, 2001; Fuentes et al, 2011). Os EGSB tem maior relação altura/diâmetro, permitindo a aplicação de velocidades superiores, podendo chegar valores de $10 \mathrm{~m} \cdot \mathrm{h}^{-1}$ ou superiores, promovendo maior contato biomassa-efluente, o que pode influenciar positivamente no aumento de eficiência do tratamento (Zaiat, 2003; Chernicharo, 1997). É composto basicamente por leito expandido, separador trifásico e recirculação. 
Um aspecto diferenciado no presente trabalho é a adição de zeólita, um aluminossilicato hidratado de metal alcalino ou alcalino terroso que, dentre suas principais características, é a sua alta capacidade de retenção de nitrogênio. Espera-se que, durante a operação do reator, esta atue na aceleração do processo de partida do reator, além de aumentar a eficiência na remoção de matéria orgânica e a quantidade de metano no biogás formado, como observado por Montalvo et al (2006).

No estudo de reatores biológicos, o estudo da hidrodinâmica tem papel importante, pois pode influenciar na velocidade das reações biológicas por meio de alterações na taxa de transferência de massa e distribuição das reações ao longo do reator (Carvalho et al, 2008).

Em geral, na caracterização hidrodinâmica de reatores, considera-se dois tipos de escoamentos ideais na modelação, o pistonado e o de mistura completa, pois para a grande maioria dos casos, estes escoamentos resultam em desempenhos diferentes e, geralmente um deles adapta-se ao processo escolhido (Levenspiel, 2000).

Na grande maioria dos trabalhos encontrados na literatura, a hidrodinâmica dos reatores EGSB não é estudada em detalhes, é considerada como mistura completa, por exemplo, nos trabalhos de Brito e Melo (1997), Fuentes et al (2011), López e Borzacconi (2011) entre outros. Trabalhos como de Bhattacharyya e Singh (2010) que buscaram detalhar melhor a hidrodinâmica do EGSB, o modelaram como um reator de fluxo pistonado com recirculação e espaço morto, com grande dispersão.

O objetivo principal do presente trabalho foi estudar, de maneira detalhada, a hidrodinâmica do reator EGSB, para que este possa ser utilizado posteriormente na modelagem matemática do funcionamento destes reatores. Além de verificar se a adição da zeólita ocorre alguma modificação no tipo de altera o escoamento do reator.

\section{MATERIAL E MÉTODOS}

O reator EGSB utilizado, cujo esquema é apresentado na Figura 1, possui volume total efetivo de 3,04 L, as dimensões do tubo, onde a biomassa mantem-se dispersa, principalmente devido à alta taxa de recirculação, e os microrganismos presentes são responsáveis pela degradação do efluente a ser tratado, são de 1,13 m de comprimento e 0,05 m de diâmetro. E, as dimensões do separador trifásico são 0,11 m de comprimento e de diâmetro. 


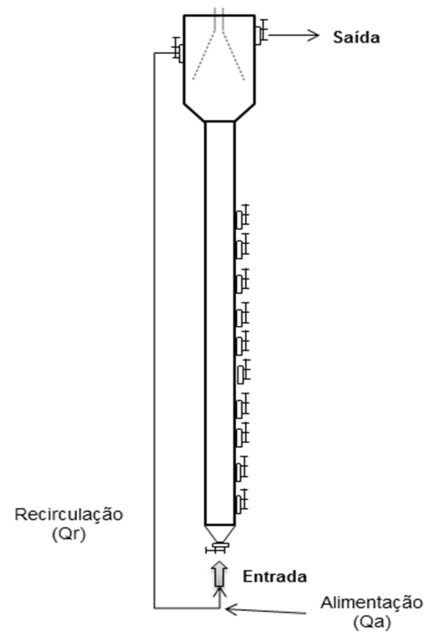

Figura 1 - Desenho esquemático do reator EGSB utilizado

O tempo de detenção hidráulica (TDH) teórico aplicado foi de 12 h, a vazão de entrada $\left(\mathrm{Q}_{\mathrm{a}}\right)$ de aproximadamente $4 \mathrm{~mL} \cdot \mathrm{min}^{-1}$ e vazão de recirculação (Qr) de aproximadamente 200 mL. min $^{-1}$, implicando em uma velocidade ascensional média de $6 \mathrm{~m} \cdot \mathrm{h}^{-1}$. A zeólita utilizada nos ensaios possui diâmetro médio de $0,15 \mathrm{~mm}$.

Foram realizados três ensaios hidrodinâmicos, sendo o primeiro somente com água (E1), o segundo com adição de $24 \mathrm{~g}$ de zeólita (E2) e o terceiro com adição de 72 g de zeólita (E3). As quantidade de zeólita adicionadas, equivalem às alturas de zeólita de 1 e $3 \mathrm{~cm}$, respectivamente, considerando o reator em repouso, ou seja, com o leito de zeólita ainda não expandido pela recirculação.

Os ensaios foram realizados pelo método estímulo-resposta, injetando $50 \mathrm{~mL}$ de traçador dextrana azul a uma concentração de 10 g. $\mathrm{L}^{-1}$. As leituras de absorbância foram realizadas em espectrofotômetro $(\lambda=650 \mathrm{~nm})$. A concentração de dextrana azul foi então obtida por meio da curva de calibração do espectrofotômetro. O tempo de duração dos ensaios foi de aproximadamente 3 vezes o tempo de detenção hidráulica.

\section{MODELAGEM MATEMÁTICA}

Para uma verificação inicial, os dados foram normalizados de acordo com Levenspiel (2000). Os valores adimensionais foram aplicados nos modelos de tanques em série (Equação 1), dispersão de pequena intensidade (Equação 2), dispersão de grande intensidade (Equação 3) e mistura completa (Equação $1 \mathrm{com} \mathrm{N}=1$ ). Sendo que $E_{\theta}$ é a distribuição de idade de saída adimensional, $\theta$ o tempo adimensional, $\sigma_{\theta}{ }^{2}$ a variância adimensional, $\mathrm{N}$ o número de tanques, e $\mathrm{Pe}_{\mathrm{p}}$ e $\mathrm{Pe}_{\mathrm{g}}$, os números de Peclet para pequena e grande dispersão, respectivamente. Os gráficos foram gerados no software Matlab 7. 


$$
\begin{aligned}
& N=\frac{1}{\sigma_{\theta}^{2}} \quad E_{\theta}=\frac{N(N \cdot \theta)^{N-1}}{(N-1) !} \cdot \exp (-N \cdot \theta) \\
& P e_{p}=\frac{2}{\sigma_{\theta}^{2}} \quad E_{\theta}=\frac{1}{2 \sqrt{\frac{\pi}{P e_{p}}} \cdot \exp \left\lfloor-\frac{P e_{p}(1-\theta)^{2}}{4}\right\rfloor} \\
& \sigma_{\theta}^{2}=\frac{2}{P e_{g}}+\frac{8}{P e_{g}{ }^{2}} \quad E_{\theta}=\frac{1}{2 \sqrt{\frac{\pi \theta}{P e_{g}}}} \cdot \exp \left[\frac{P e_{g}(1-\theta)^{2}}{4 \theta}\right\rfloor
\end{aligned}
$$

Como o diâmetro do separador $(0,11 \mathrm{~m})$ é relativamente maior que no tubo $(0,05 \mathrm{~m})$, causando mudança significativa na velocidade ascensional, optou-se por dividir o reator em dois reatores tubulares em série. O número de Peclet foi ajustado seguindo o critério de menor soma dos erros ao quadrado (método dos mínimos quadrados), utilizando a ferramenta fminsearch do Matlab 2011.

O modelo tubular é um exemplo de modelo a parâmetros distribuídos, nos quais se admite que as propriedades variem com as coordenadas espaciais, ou seja, ao longo do reator (Pinto; Lage, 2001; Tavares, 2008).

O modelo de reator tubular e as condições de contorno consideradas estão descritas nas Equações 4, 5 e 6, onde v é a velocidade ascensional, C a concentração de dextrana na saída do reator, $\mathrm{D}$ o coeficiente de dispersão axial, $\mathrm{C}_{\text {in }}$ a concentração de entrada, $\mathrm{t}$ o tempo ao longo do comprimento (L) do reator.

$$
\begin{aligned}
& \frac{\partial C}{\partial t}=-v \frac{\partial C}{\partial z}+D \frac{\partial^{2} C}{\partial z^{2}} \\
& \operatorname{Em} z=0 ; D \frac{\partial C}{\partial z}=v\left(C_{1}-C_{i n}\right) \\
& \operatorname{Em} z=L ; \frac{\partial C}{\partial z}=0
\end{aligned}
$$

$\mathrm{Na}$ resolução das equações, foi utilizado o método das diferenças finitas centrais de ordem 2, cujas aproximações de primeira e segunda ordem estão descritas nas Equações 7 e 8 , respectivamente. O objetivo deste método é transformar um problema de equações diferenciais em um problema de equações algébricas, através da discretização do domínio da variável independente, dividindo o domínio de cálculo em subdomínios e em seguida gerando aproximações para as derivadas dependentes (Pinto e Lage, 2001; Fontoura, 2004).

$$
\begin{aligned}
& \frac{\partial C}{\partial z}=\frac{C_{i+1}-C_{i-1}}{2 d z} \\
& \frac{\partial^{2} C}{\partial z^{2}}=\frac{C_{i-1}-2 C_{i}+C_{i+1}}{d z^{2}}
\end{aligned}
$$


Em um sistema convectivo-difusivo, o número de Peclet $(\mathrm{Pe})$ expressa a relação entre a convecção e a difusão, sendo que quanto maior o $\mathrm{Pe}$, maior a influência da do processo convectivo para o transporte de massa. E quando o Pe é muito baixo, o processo é tratado como um processo difusivo (Franceschini, 2002). No presente trabalho, o valor de Peclet, expresso pela Equação 9 foi o parâmetro de ajuste.

$$
P e=\frac{v \cdot L}{D}
$$

No modelo proposto, o reator foi dividido em duas regiões, dois reatores tubulares em série, sendo uma a região do tubo e outra a do separador. O comprimento de cada região foi dividido em $\mathrm{N}$ valores de $\Delta \mathrm{z}$, e as equações resolvidas utilizando a ferramenta ode $15 \mathrm{~s}$ do programa Matlab 2011. O número de Peclet foi ajustado para cada uma das regiões utilizando o método dos mínimos quadrados.

\section{RESULTADOS E DISCUSSÃO}

Na Tabela 1 têm-se os valores de parâmetros obtidos para os testes preliminares, apresentando o número de reatores calculados para tanques em série $(\mathrm{N})$, os valores de Peclet para os modelos de pequena $\left(\mathrm{Pe}_{\mathrm{p}}\right)$ e grande dispersão $\left(\mathrm{Pe}_{\mathrm{g}}\right)$, e os valores do coeficiente de correlação (r) para cada modelo. E na Figura 2 estão apresentadas as curvas dos dados experimentais e dos modelos testados para os três ensaios.

Tabela 1 - Valores obtidos durante os testes dos modelos hidrodinâmicos

\begin{tabular}{c|c|c|c|c|c|c|c}
\cline { 2 - 7 } & \multicolumn{2}{|c|}{ Tanques em Série } & \multicolumn{2}{c|}{ Pequena Dispersão } & \multicolumn{2}{c|}{ Grande Dispersão } & $\begin{array}{c}\text { Mistura } \\
\text { Completa }\end{array}$ \\
\hline Zeólita & $\mathbf{N}$ & $\mathbf{R}$ & $\mathbf{P e}_{\mathbf{p}}$ & $\mathbf{r}$ & $\mathbf{P e}_{\mathbf{g}}$ & $\mathbf{r}$ & $\mathbf{r}$ \\
\hline--- & 1,42 & 0,808 & 2,84 & 0,642 & 5,08 & 0,331 & 0,916 \\
\hline $1 \mathrm{~cm}$ & 1,55 & 0,893 & 3,11 & 0,709 & 5,41 & 0,433 & 0,905 \\
\hline $3 \mathrm{~cm}$ & 1,78 & 0,882 & 3,56 & 0,702 & 5,95 & 0,393 & 0,873 \\
\hline
\end{tabular}

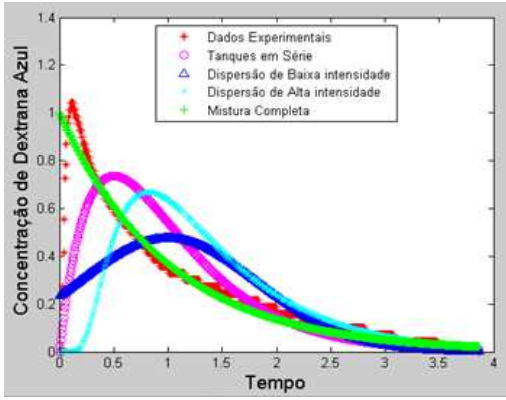

(a)

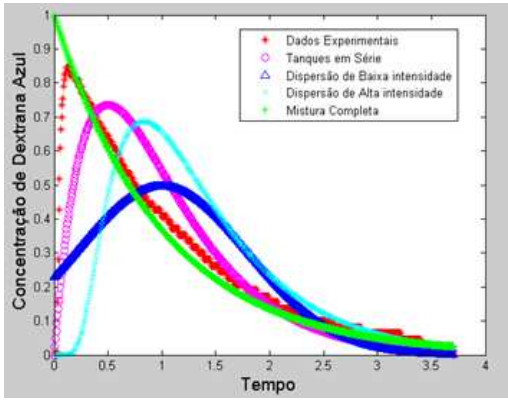

(b)

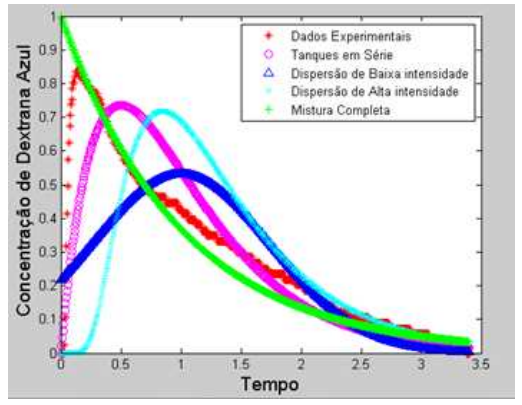

(c)

Figura 2 - Curvas adimensionais dos dados experimentais e dos modelos de mistura completa, tanques em série, pequena e grande dispersão (a) sem adição de zeólita e com leito de zeólita de (b) $1 \mathrm{~cm}$ e (c) $3 \mathrm{~cm}$ 
Observa-se que com a adição de zeólita o número de Peclet aumenta, indicando que a adição da zeólita afeta, sensivelmente, o escoamento do reator diminuindo a dispersão no mesmo. Em relação ao modelo de taques em série, nota-se o valor de $\mathrm{N}$ calculado está entre 1 e 2, aproximando-se mais de $\mathrm{N}=2$ conforme aumenta-se a quantidade de zeólita.

Este teste foi realizado para se conhecer a tendência dos dados. No entanto, os modelos utilizados não consideram o efeito da recirculação. Com o intuito de conhecer mais detalhadamente a hidrodinâmica do EGSB, testou-se o ajuste ao modelo de escoamento tubular com dispersão. Através do ajuste do número de Peclet, pode-se concluir qual fenômeno, convectivo ou difusivo, predomina nas duas partes do reator (separador e tubo).

Os valores de Peclet obtidos nos três ensaios foram muito similares. Para a região do tubo os valores obtidos foram 5,08 (E1); 5,03 (E2) e 4,86 (E3). Na região do separador, os valores foram próximos a zero, apresentando valores de na ordem de $10^{-6}$ nos três ensaios.

Nos três ensaios realizados, o separador apresentou valor de Pe muito próximo a zero, implicando em uma predominância do processo difusivo, podendo-se desconsiderar que comporta-se como um reator CSTR. O tubo apresentou valores baixos de Pe, comportando-se como um reator tubular com alta dispersão, como observado também no trabalho de Bhattacharyya e Singh (2010). As curvas experimentais e do modelo de dois reatores tubulares com dispersão em série estão apresentadas na Figura 3.

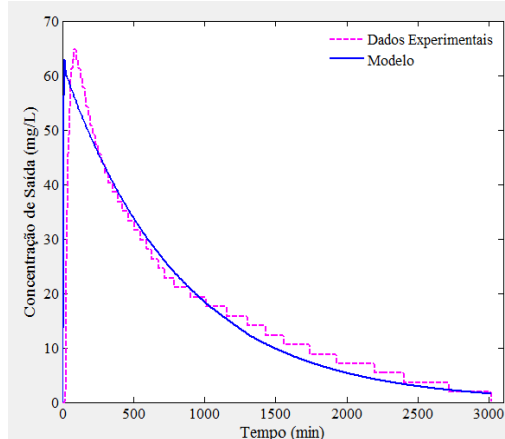

(a)

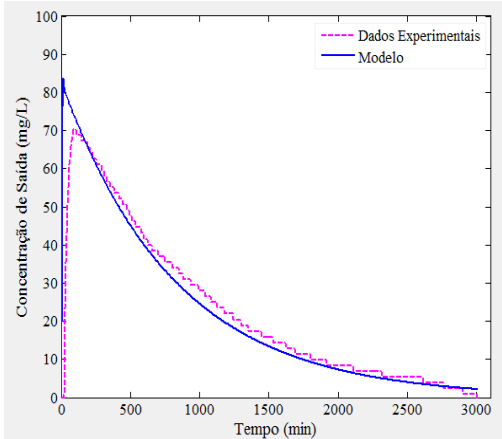

(b)

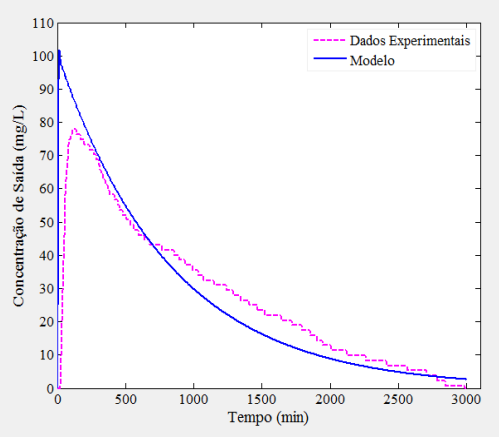

(c)

Figura 3 - Curvas experimentais e do modelo de dois reatores tubulares com dispersão em série para os ensaios (a) sem adição de zeólita (E1), e com leito de zeólita de (b) $1 \mathrm{~cm}$ (E2) e (c) $3 \mathrm{~cm}$ (E3)

A soma dos erros ao quadrado nos três ensaios apresentou valores na ordem de $10^{4}$, com coeficiente de correlação de 0,94 para E1, 0,93 para E2 e, 0,89 para E3.

De acordo com Secchi (1995), quanto mais rigorosa a descrição de um processo maior o número de equações resultantes e, mais difícil o tratamento destas. Assim, embora elas possam ser resolvidas, é aconselhável o uso de aproximações, a fim de reduzir as equações e diminuir a complexidade das mesmas. Sugere-se que uma nova modelagem seja realizada, considerando as duas regiões do EGSB como uma série de CSTR, por exemplo. 


\section{CONCLUSÃO}

Buscou-se conhecer a hidrodinâmica do reator EGSB de maneira mais detalhada, considerando a influência da recirculação, e não somente tratando-o como um modelo onde somente entrada e saída são consideradas (modelo caixa preta).

O modelo de dois reatores tubulares em série mostrou, por meio do ajuste do número de Peclet, que o reator pode ser descrito como um reator tubular com alta dispersão (região do tubo), seguido de um reator de mistura completa (região do separador).

A adição de zeólita ao reator apresentou pequena influencia no escoamento do reator, diminuindo, sensivelmente a difusão no tubo com o aumento na quantidade da mesma.

\section{AGRADECIMENTOS}

Agradecemos a Capes concessão da bolsa de doutorado e a FAPESP pelo apoio ao Projeto Temático nº 2009/15984-0.

\section{REFERÊNCIAS}

BHATTACHARYYA, D.; SINGH, K. S. Understanding the Mixing Pattern in an Anaerobic Expanded Granular Sludge Bed Reactor: Effect of Liquid Recirculation. J. Environ. Eng. v. 136, p. 576-584, 2010.

BRITO, A. G.; MELO, L. F. A Simplified Analysis of Reaction and Mass Transfer in UASB and EGSB Reactors. Environ. Tech. v.18, p. 35-44, 2010.

CARVALHO, K. Q; SALGADO, M. T.; PASSIG, F. H; PIRES, E. C. Avaliação hidrodinâmica de reator UASB submetido à variação cíclica de vazão. Eng. Sanit. Ambient. v.13, n.2, p. 226-235, 2008.

CHERNICHARO, C. A .L. Reatores anaeróbios. DESA-UFMG, Belo Horizonte, 245 p. 1997.

FONTOURA, D. V. R. Contribuição à modelagem matemática do reator anaeróbio horizontal de leito fixo (RAHLF) para tratamento de águas residuárias. 131f. Dissertação (Mestrado em Engenharia Química). Universidade Federal de São Carlos - UFSCar, São Carlos, 2004.

FRANCESCHINI, F. S. Modelagem Mecanica e Aproximação por Métodos Estabilizados de Escoamentos Multicomponentes. Dissertação (Mestrado em Engenharia Mecânica). Escola de Engenharia da Universidade Federal do Rio Grande do Sul, 2002.

FUENTES, M.; SCENNA, N. J.; AGUIRRE, P. A. A coupling model for EGSB bioreactors: Hydrodynamics and anaerobic digestion processes. Chem. Eng. Proc. v.50, p. 316-324, 2011.

KATO, M. T.; FLORENCIO, L; ARANTES, R. F. M. Tratamento de Esgotos Sanitários em Reatores Tipo EGSB Anaeróbio e Aeróbio. Coletânea de trabalhos técnicos - Pós-tratamento de efluentes de reatores anaeróbios, PROSAB. v. 2, p. $165-174,2001$. 
LEVENSPIEL, O. Engenharia das reações químicas. 3. Ed. São Paulo: Edgard Blucher, 2000.

LÓPEZ, I.; BORZACCONI, L. Modelling of an EGSB treating sugarcane vinasse using first-order variable kinetics. Water Science \& Technology, 2011.

MONTALVO, S.; GUERRERO, L.; BORJA, R.; TRAVIESO, L.; SÁNCHEZ, E.; DÍAZ, F. Use of natural zeolite at different doses and dosage procedures in batch and continuous anaerobic digestion of synthetic and swine wastes. Resourc., Conserv. Recyc. v. 47, p. 26-41, 2006.

PINTO, J. C.; LAGE, P. L. C. Métodos numéricos em problemas de engenharia química. Série: Escola Piloto em Engenharia Química COPPE/UFRJ. 1. ed. Rio de Janeiro: E-papers, 2001.

SECCHI, A. R. Modelagem e Simulação de Processos. Notas de Aula. Escola de Engenharia - Universidade Federal do Rio Grande do Sul, 1995.

TAVARES, A. C. Modelagem matemática da degradação da glicose, com produção de hidrogênio, em reatores anaeróbios de leito fixo. 2008. 85f. Dissertação (Mestrado em Engenharia Hidráulica e Saneamento), Escola de Engenharia, USP, São Carlos, 2008.

ZAIAT, M. Desenvolvimento e análise de biorreatores anaeróbios contendo células imobilizadas para tratamento de águas residuárias: reator anaeróbio horizontal de leito fixo, reator anaeróbio operado em bateladas sequenciais. Texto (Livre Docência). Escola de Engenharia de São Carlos - USP, 2003. 